\title{
Venous Mapping of Vascular Malformations using Cranial 4D Flow MRI
}

Grant Roberts ${ }^{1}$, Michael Loecher ${ }^{2}$, Leonardo Rivera-Rivera ${ }^{3}$,

Patrick Tursk $i^{3}$, Kevin Johnson ${ }^{1,3}$, Oliver Wieben ${ }^{1,3}$, and Laura Eisenmenger ${ }^{3}$

THE UNIVERSITY

WISCONSIN

MADISON

Departments of ${ }^{1}$ Medical Physics and ${ }^{3}$ Radiology, University of Wisconsin - Madison; Department of ${ }^{2}$ Radiology, Stanford University

\section{Purpose}

- Management of intracranial vascular lesions such as arteriovenous malformations (AVMs), vein of Galen malformations (VGMs), and dural arteriovenous fistulas (DAVFs) can be complex with significant morbidity and mortality.

- Endovascular intervention via a venous approach, or trans-venous embolization (TVE), is commonly used to treat VGMs and DAVFs.

- TVE for AVM treatment is now also gaining interest ${ }^{1}$.

- Accurate characterization of venous drainage is essential to a TVE treatment approach.

- Venous mapping can be limited on digital subtraction angiography due to mixing of non-opacified blood.

- Purpose: Present a novel approach to perform comprehensive venous mapping of flow patterns through retrospective 'virtual injection' applied to high resolution 4D Flow MRI.

\section{Methods}

- 4D flow data was acquired on clinical 3T scanners with a radiallyundersampled PCVIPR ${ }^{2}$ acquisition.

- Whole brain coverage, $6 \mathrm{~min}$. scan, VENC $=80 \mathrm{~cm} / \mathrm{s}, 0.7 \mathrm{~mm}$ res.

- The 4D flow data were used to (1) generate high-resolution angiograms, (2) select seeding locations to initiate 'virtual injections', which were (3) calculated from the acquired velocity vector field, and (4) visualized as streamlines.

- Processing included stochastic noise corrections, displacement corrections $^{3}$, and fluid constraints to track arterial and venous blood flow in areas of interest ${ }^{4}$.

- For comparisons, velocity selective ASL was also acquired.

\section{Findings}

- The new approach was in good qualitative agreement with PCASL with added advantages of (1) retrospective choice of 'injection site' enabling (2) venous capture with (3) anterograde and retrograde blood flow evaluation.

- For example, a portion of a shunt or nidus that is drained by each vein can be visualized.

- This may prove essential in appropriate selection of embolization candidates and pre-procedure planning.

- Figure $1 \mathrm{~b}-\mathrm{c}$ shows a case with streamline generation seeds placed distal to the AVM nidus.

○ Retrograde tracking shows the nidus supplied by each vein.

\section{Summary}

- Probabilistic streamlines from corrected 4D flow MR data allow for complex arterial and venous mapping in vascular malformations without being affected by mixing of non-opacified blood.

- This approach provides valuable new insights into pre-procedural planning with guidance for selective embolization.

- Future studies are needed to assess the impact on improved preprocedure planning and patient outcomes.

\section{References}

[1] O. Choudhri et al. Neurosurgery, vol. 77, no. 4, pp. 644-652, 2015.

[2] T. Gu et al. AJNR Am J Neuroradiol, vol. 26, no. 4, pp. 743-749, 2005.

[3] D. A. Steinman et al. J Magn Reson Imaging, vol. 7, no.

[4] M. Loecher et al. Society for Magnetic Resonance Angiography, 2014.

\section{D Flow Venous Tracking}

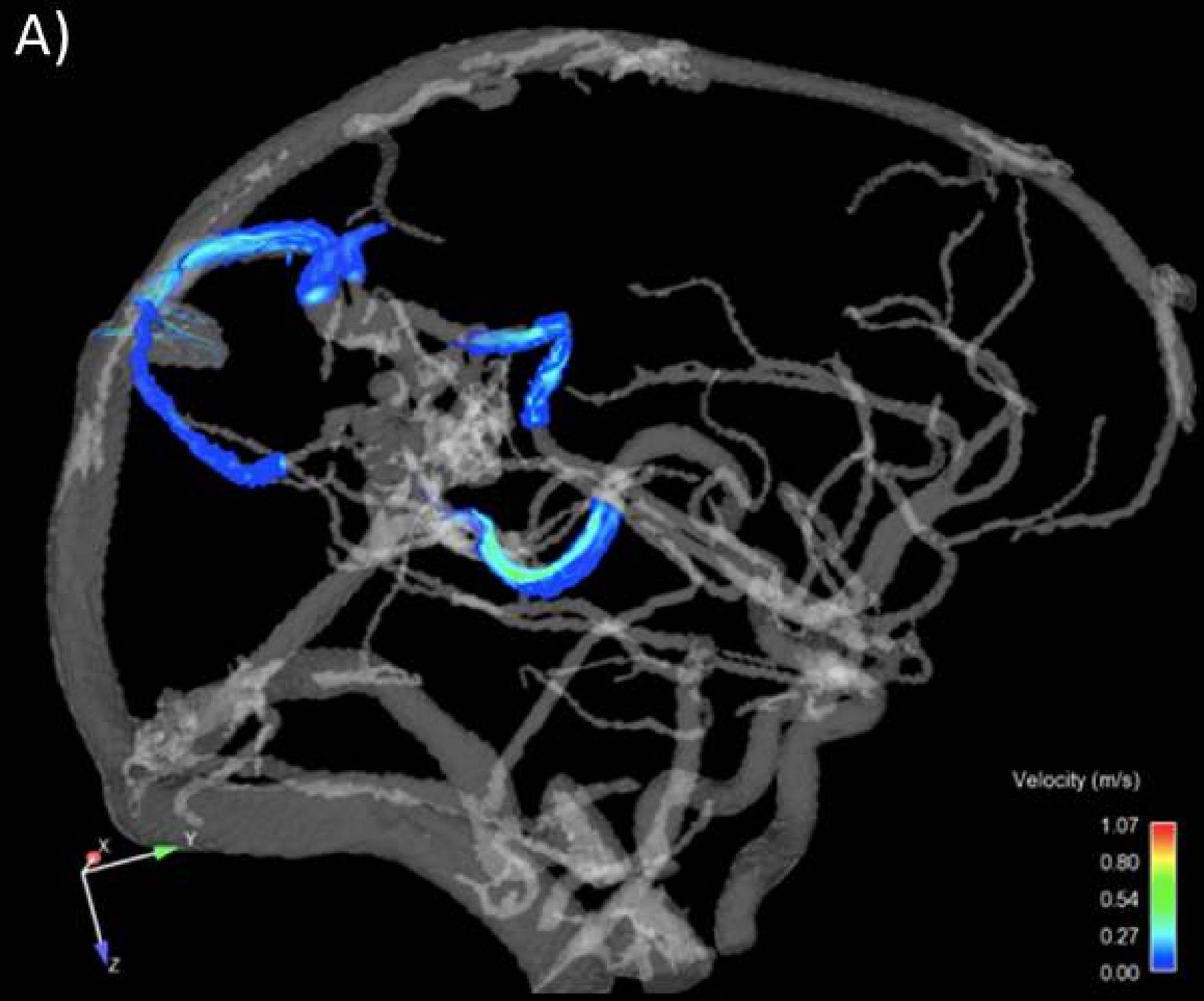

4D Flow angiogram and velocity maps of left transverse sinus DAVF.
B)

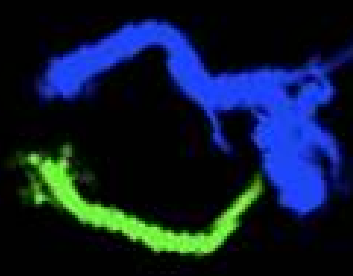

Seeds placed distal to nidus in the two draining veins.

C)

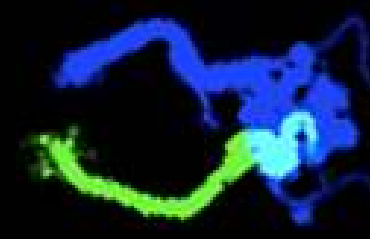

Retrograde flow tracking through nidus with faster flow through draining vein (blue) and quicker filling of the feeding artery.

D)

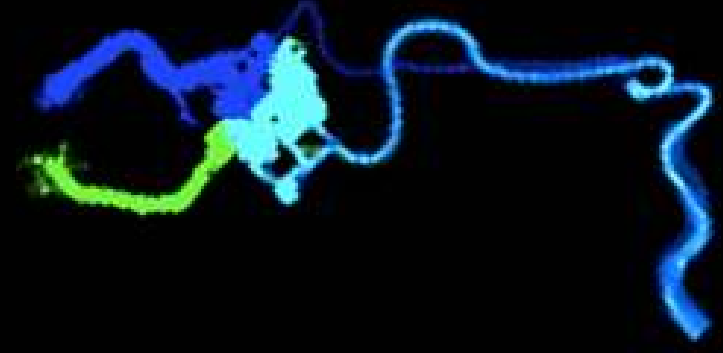

Flow tracking showing the portion of the nidus draining into the slower vein (green) reaching the feeding artery. 